\title{
Glutamatergic Projections from the Entorhinal Cortex to Dorsal Dentate Gyrus Mediate Context-Induced Reinstatement of Heroin Seeking
}

\author{
Feifei Ge', Na Wang', Cailian Cui*,', Yijing Li', Yan Liu', Yaoying Ma', Shuli Liu', Haolin Zhang' and \\ Xiaowei Sun' \\ 'Department of Neurobiology, School of Basic Medical Sciences, Key Laboratory for Neuroscience of the Ministry of Education and National Health \\ and Family Planning Commission, Neuroscience Research Institute, Peking University, Beijing, China
}

\begin{abstract}
Reexposure to the context associated with heroin intake provokes relapse to drug taking after abstinence. The dorsal dentate gyrus (dDG) and entorhinal cortex (EC) have been implicated in contextual memory processing, but the underlying circuit mechanisms in contextinduced relapse remain poorly understood. In this study, using a self-administration rat model, we found that activation and synaptic transmission of glutamatergic projections from the $\mathrm{EC}$ to the upper blade of dentate gyrus $\left(\mathrm{dDG}_{\mathrm{ub}}\right)$ were significantly enhanced during context-induced reinstatement of heroin seeking. This effect was associated with increased of phosphorylation of GluN2B-containing NMDA receptors (GluN2B) at Y I472, ratio of GluN2B membrane/total protein levels, and expression of downstream extracellular signalregulated kinase-I/2 (ERKI/2) in the $\mathrm{dDG}$ region. Furthermore, DREADD-mediated specific inactivation of the EC-dDG pathway or disconnection of the pathway with local postsynaptic GluN2B-ERKI/2 signaling both decreased context-induced reinstatement of heroin seeking. These experimental manipulations had no effect on saccharin-reinforced responding and general locomotor activity in rats. Our results indicate that the EC-dDG pathway mediates context-induced reinstatement of heroin seeking, via the activation of postsynaptic GluN2B-ERKI/2 signaling in the dDG.

Neuropsychopharmacology (2017) 42, I860-1870; doi:I0.1038/npp.2017.I4; published online I5 February 2017
\end{abstract}

\section{INTRODUCTION}

Reexposure to an environmental context associated with the intake of heroin can provoke drug craving and subsequent relapse, even after prolonged abstinence periods (O'Brien et al, 1992; Wikler, 1973). Rat studies using an ABA renewal procedure (Crombag et al, 2008) have shown that reexposure to a previous heroin-paired context after extinction can reinstate heroin-seeking behavior (Bossert et al, 2011; Bossert et al, 2012). The results of Bossert et al (2012) showed that the projections from ventral medial prefrontal cortex (vmPFC) to nucleus accumbens (NAc) shell play a role in context-induced reinstatement of heroin seeking. These authors also demonstrated that the projections from ventral subiculum (vSub) to NAc shell but not vmPFC contribute to context-induced reinstatement of heroin seeking (Bossert et al, 2016). There is also evidence that the Src family kinasemediated GluN2B activation in the dorsal hippocampus is necessary for memory processes that promote context-

* Correspondence: Professor C Cui, Department of Neurobiology, Neuroscience Research Institute, Peking University, 38 Xueyuan Road, Beijing I00 19I, China, Tel: +86 I0 8280 I 120, Fax: +86 I0 8280 II20, E-mail: clcui@bimu.edu.cn

Received 2 March 2016; revised 10 January 2017; accepted II January 2017; accepted article preview online 20 January 2017 induced reinstatement of cocaine seeking (Xie et al, 2013; Ramirez et al, 2009).

Relapse to drug seeking requires memory recall. It is well known that the dorsal hippocampus is critical for this memory process (Kim and Fanselow, 1992). Previous studies showed that the dorsal dentate gyrus (dDG) encodes contextual information of both fear (Redondo et al, 2014) and reward (Hernandez-Rabaza et al, 2008), and it is activated during retrieval of morphine-associated contextual memories (Rivera et al, 2015). These lines of evidence point to the $\mathrm{dDG}$ as a potential brain area that plays a role in contextual memories whose activation promotes contextinduced reinstatement of heroin seeking.

Glutamatergic neurotransmission in the dorsal hippocampus has been demonstrated to be necessary for drug contextinduced reinstatement of cocaine seeking (Xie et al, 2010). The $\mathrm{dDG}$ receives glutamatergic input from the $\mathrm{EC}$ through the perforant pathway (PP) (Deng et al, 2010), and the EC plays a role in both spatial and nonspatial memory processes (Van Cauter et al, 2013). In addition, conditioned context learning can evoke lasting changes to the synaptic strength in PP-dDG (Carretero-Guillen et al, 2013) and there is evidence that neuronal activity in the DG is mainly dependent on GluN2Bmediated neurotransmission (Ge et al, 2007).

In the present study, we hypothesized that glutamatergic projections from the EC to dDG mediate context-induced 
reinstatement of heroin seeking, and it is also modulated by GluN2B subunits and the downstream signaling in the dDG region. To address these questions, we first investigated the role of glutamatergic projections from the EC to dDG in context-induced reinstatement of heroin seeking. Next, we examined the alteration of synaptic transmission from the EC to dDG, GluN2B membrane expression, phosphorylation level of GluN2B, and subsequent extracellular signalregulated kinase-1/2 (ERK1/2) signaling in the dDG. Finally, we determined the effect of inhibiting the EC-dDG pathway or inactivating GluN2B-ERK1/2 signaling in the $\mathrm{dDG}$ on context-induced reinstatement of heroin seeking.

\section{MATERIALS AND METHODS}

\section{Animals}

Male Sprague-Dawley rats were individually housed in a temperature- and humidity-controlled vivarium on a reversed $12 \mathrm{~h}$ light-dark cycle. All experimental procedures were approved by the Animal Use Committee of Peking University Health Science Center.

\section{Procedures}

Experiment 1: activation of glutamatergic neurons in the $d D G$ mediated context-induced reinstatement of heroin seeking. Rats received heroin self-administration training in context A, followed by extinction training in context B. After reexposure to context $\mathrm{A}$ or $\mathrm{B}$ for $90 \mathrm{~min}$, the rats $(n=4$ per group) were anesthetized and brain sections were prepared for immunofluorescence. Other rats were implanted with cannulas to the $\mathrm{dDG}$ ( $n=10$ per group) or $\mathrm{vDG}$ ( $n=8-9$ per group) during the extinction training. On the test day, the rats were microinjected with a mixture of baclofen and muscimol (B $+\mathrm{M}, \mathrm{GABA}_{\mathrm{B}}$ and $\mathrm{GABA}_{\mathrm{A}}$ receptor agonists, respectively, $250 \mathrm{ng} / \mu \mathrm{l}, 0.5 \mu \mathrm{l}$ per side) (Keiflin et al, 2013) or saline $(0.5 \mu \mathrm{l}$ per side) 5-10 min before reexposure to context A. At $72 \mathrm{~h}$ after the reinstatement test, the rats were trained for saccharin-reinforced behavior. Assignment of rats to treatment groups and vehicle/drug order were counterbalanced.

Experiment 2: heroin-paired context exposure induced potentiation of synaptic transmission from the EC to the $d D G$. Rats were injected with Fluoro Gold (FG, a retrograde tracer, $4 \%, 1 \mu \mathrm{l})$ (Weiner, 2008) in the $\mathrm{dDG}(n=4$ per group) unilaterally. After reexposure to context A or B for 90 min, we detected the expression of FG in the EC. The excitability of the EC-dDG pathway was examined in vivo using electrophysiology in anesthetized rats ( $n=8$ per group) immediately after reexposure to context A or B for $60 \mathrm{~min}$. Electrophysiological procedures were performed as previously described (Hu et al, 2014).

Experiment 3: inhibition of EC-dDG projections decreased context-induced reinstatement of heroin seeking with $D R E A D D s$. At 2 weeks before the heroin self-administration training, rats were anesthetized with sodium pentobarbital and given virus injections ( $A A V_{8}-h S y n-h M 4 d$ (Gi)$m$ Cherry or $A A V_{5}$-Syn-mCherry; $1 \mu \mathrm{l}$ per side; Vector Core, University of North Carolina) bilaterally into the EC ( $n=11$ per group). The injections were given over $5 \mathrm{~min}$, using an infusion pump, and left in place for $10 \mathrm{~min}$ (Mahler et al, 2014). Guide cannulas were implanted bilaterally into the dDG 1 week before the reinstatement testing. For all behavioral experiments, $5 \mathrm{~min}$ before each test, rats were given bilateral intra-dDG microinjections of a vehicle (saline) or CNO ( $1 \mathrm{mM}, 0.3 \mu \mathrm{l}$ per side; Tocris; 4936 ) at a rate of $0.15 \mu \mathrm{l} / \mathrm{min}$ and left in place for an additional $1 \mathrm{~min}$. At the end of behavioral testing, cannula placements and hM4Di receptor expression were evaluated with immunofluorescence.

Experiment 4: reexposure to the heroin-paired context elevated the GluN2B-ERK1/2 signaling in the dDG. The rats were decapitated immediately after reexposure to context A or B for $30 \mathrm{~min}$ and the brains were quickly retrieved and homogenized. We detected the total and membrane protein fractions of AMPAR (GluA1 and GluA2) and NMDAR (GluN1, GluN2A, and GluN2B) receptors ( $n=7$ per group) and the phosphorylation level of GluN2B and ERK1/2 ( $n=7$ per group) in the dDG by western blot.

Experiment 5: inhibition of EC-dDG projections via GluN2B-ERK1/2 signaling decreased context-induced reinstatement of heroin seeking. There were three groups of rats: control, contralateral, and ipsilateral groups ( $n=10$ per group). $5-10 \mathrm{~min}$ before the reinstatement testing, the rats in control group were injected with saline in unilateral dDG and contralateral/ipsilateral EC. Rats in contralateral or ipsilateral group were injected with PP2 (Xie et al, 2013) (Src family kinase inhibitor, $6.25 \mathrm{ng} / 0.5 \mu \mathrm{l}$ per side) in unilateral $\mathrm{dDG}$ and $\mathrm{B}+\mathrm{M}$ in contralateral or ipsilateral EC. After the $60 \mathrm{~min}$ reinstatement testing, the brains were quickly dissected and frozen for future western blot assays. In addition, RO25-6981 (Shen et al, 2016) (GluN2B-selective antagonist, $1 \mu \mathrm{g} / 0.5 \mu \mathrm{l}$ per side) and $\mathrm{B}+\mathrm{M}$ disconnection ( $n=9$ per group), same as U0126 (Xu et al, 2012) (MEK1/2 inhibitor, $1 \mu \mathrm{g} / 0.5 \mu \mathrm{l}$ per side) and $\mathrm{B}+\mathrm{M}$ disconnection ( $n=7$ per group) were also tested as described above.

\section{Statistical Analysis}

Data are expressed as mean \pm SEM. Data from FG-Fos doublepositive neuron counts (Figure $2 \mathrm{c}$ ) and immunoblotting in the PP2 and $\mathrm{B}+\mathrm{M}$ disconnection treatments (Figure $5 \mathrm{~b}$ and $\mathrm{c}$ ) were analyzed with ANOVA, followed by Tukey's or Bonferroni's post hoc test. When two groups were compared, the data were statistically analyzed using a two-tailed unpaired $t$-test. A two-way ANOVA, followed by Bonferroni's multiple comparisons test, was used for analyzing other data. Between- and within-subject factors used in the analysis are described in the text. The data were processed by the software Graph Pad Prism 6.0 (GraphPad). Statistical significance was set at $P<0.05$.

For detailed methodologic information, please see Supplementary Materials and Methods.

\section{RESULTS}

\section{The dDG Is Necessary to Context-Induced Reinstatement of Heroin Seeking}

In this study, rats received intravenous heroin selfadministration training in context $\mathrm{A}$ followed by extinction 
Self-administration Training

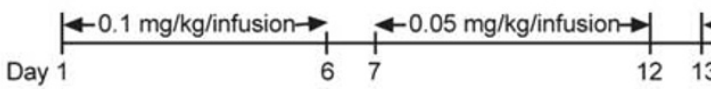

b

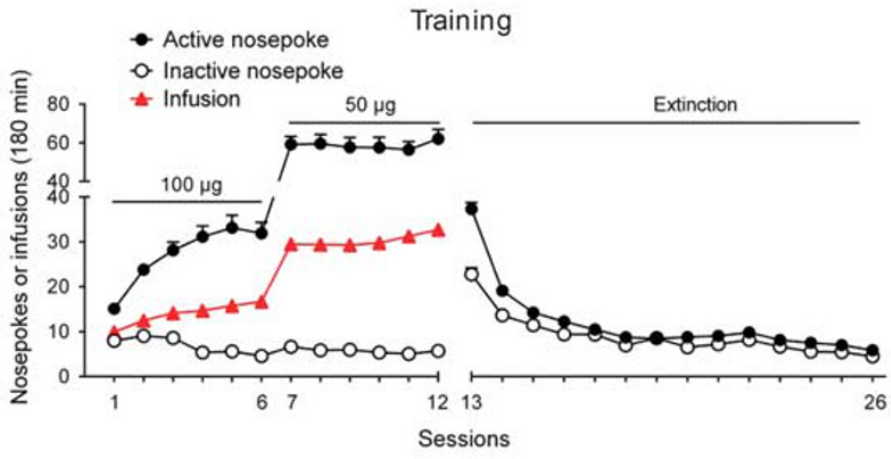

C

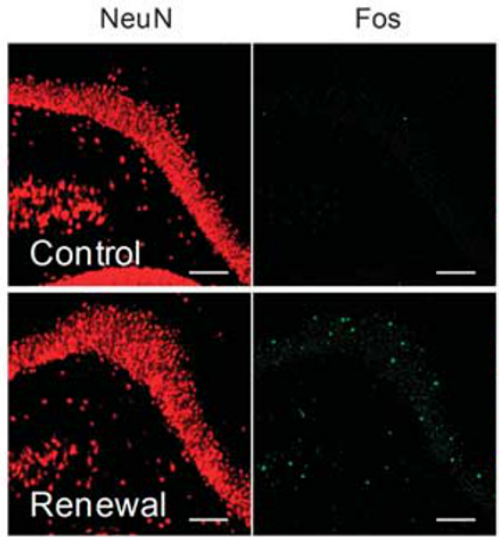

e

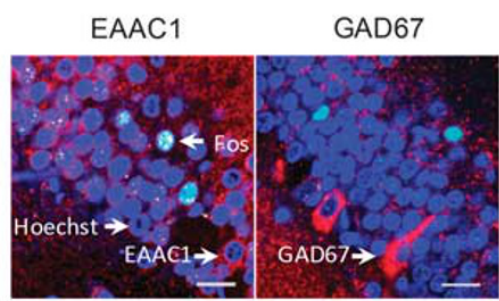

Merge

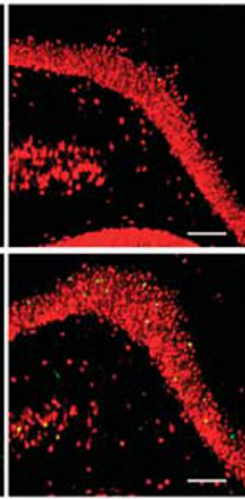

f

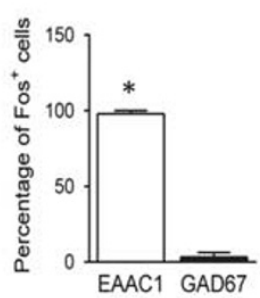

Reinstatement
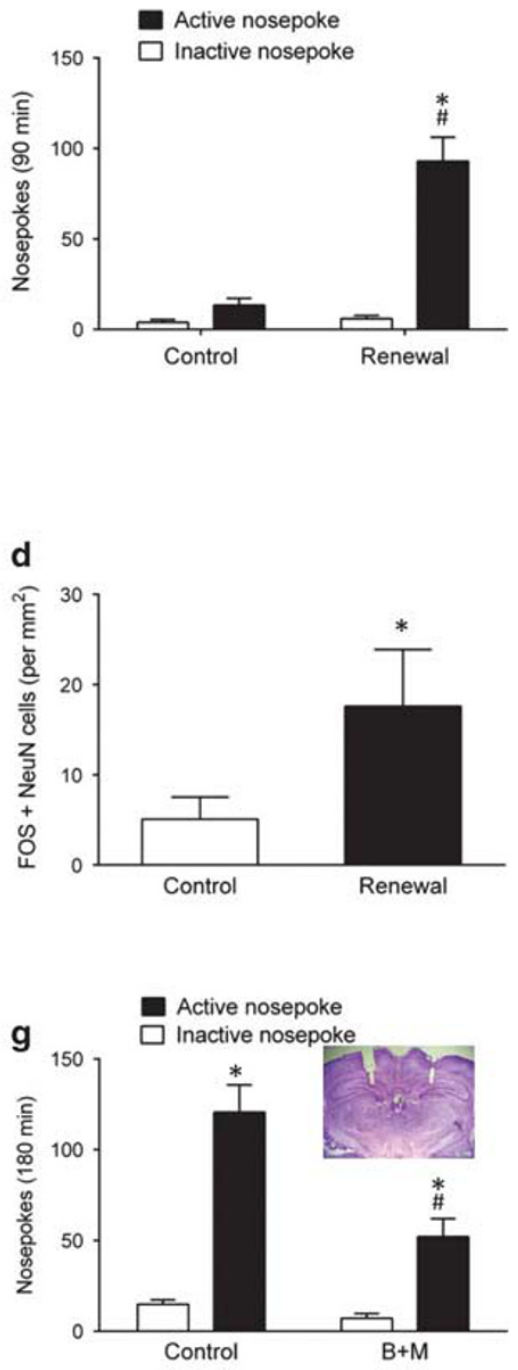
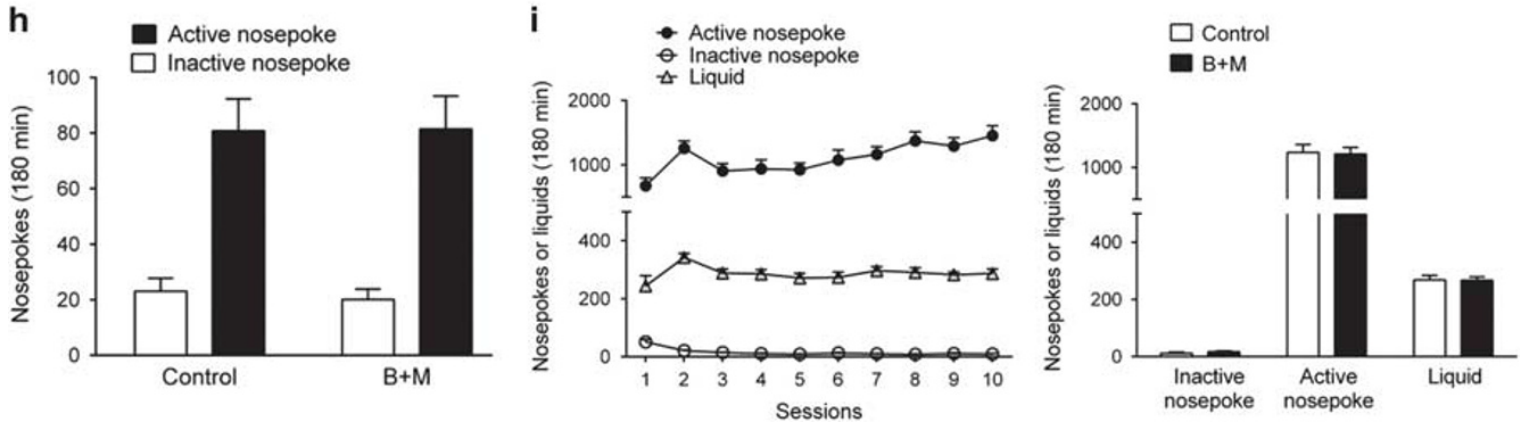
training in context B (Bossert et al, 2011) (Figure 1a), and then reexposed to context $\mathrm{A}$ ( $\mathrm{ABA}$, renewal) or $\mathrm{B}(\mathrm{ABB}$, control) to carry out the reinstatement testing. During the reinstatement testing, there was a significant difference between the active and inactive nose-pokes in the renewal group, and the number of active nose-pokes in the renewal group was higher than that in the control group. These results indicate that the heroin-seeking behavior was reinstated when rats were reexposed to the heroin-paired context (Figure 1b). The statistical analysis showed a significant interaction between Group and Nose-Poke $\left(\mathrm{F}_{(1,14)}=28.62, P=0.0001\right)$. Next, we measured the number of Fos and NeuN double-positive cells (per $\mathrm{mm}^{2}$ ) in the $\mathrm{dDG}$ with fluorescence immunohistochemistry after reexposure to context A or B for 90 min. The number of Fos-NeuN doublepositive cells (per $\mathrm{mm}^{2}$ ) in the renewal group was $17.6 \pm 1.7$, and this was 3.5 times higher than the control $(5.1 \pm 0.7)$ (Figure $1 \mathrm{c}$ and $\mathrm{d}$ ). The Fos-positive neurons in the dDG were located mainly in the $\mathrm{dDG}_{\mathrm{ub}}$ and only sparsely in the $\mathrm{dDG}_{\mathrm{lb}}$. Moreover, these Fos-positive nuclei were colabeled with EAAC1 (glutamate transporter EAAC1, a marker of glutamatergic neurons) (Lammel et al, 2012), but not GAD67 (Kaufman et al, 1986) in the renewal group, indicating that the glutamatergic neurons in the $\mathrm{dDG}_{\mathrm{ub}}$ were selectively excited by reexposure to the heroin-paired context (Figure $1 \mathrm{e}$ and $\mathrm{f}$ ). To determine whether the $\mathrm{dDG}_{\mathrm{ub}}$ activation mediates context-induced reinstatement, $B+M$ was microinfused bilaterally into the $\mathrm{dDG}$ to inactivate the neurons in the dDG. Results showed bilateral inactivation of $\mathrm{dDG}$ decreased context-induced reinstatement of heroin seeking (Figure 1g). The analysis showed a significant interaction between Drug and Nose-Poke $\left(F_{(1,18)}=11.02\right.$, $P=0.0038)$. There was no difference observed in saccharinreinforced responding with $\mathrm{B}+\mathrm{M}$ treatment (Figure 1i). In addition, bilateral inactivation of ventral DG (vDG) had no effect on context-induced reinstatement of heroin seeking (Figure 1h). Thus, the results shown in Figure 1 demonstrated that the activation of glutamatergic neurons in the $\mathrm{dDG}$ region mediates context-induced reinstatement of heroin seeking.

\section{EC Glutamatergic Projections to the dDG Mediate Context-Induced Reinstatement of Heroin Seeking}

To determine the function of afferents projecting to the $\mathrm{dDG}$ in mediating context-induced reinstatement, we infused FG unilaterally to the dDG (Figure 2a). Then, we measured the FG-Fos double-positive neurons in the EC by triple staining with FG, Fos, and NeuN. Consistent with recent results (Knierim et al, 2014), our data showed that local injection of FG in the dDG is retrogradely traced to the EC. The number of FG-Fos double-positive neurons (per $\mathrm{mm}^{2}$ ) in the renewal group was $97.6 \pm 10.7$, and this was significantly higher than the naive and control groups $(9.7 \pm 2.4$ and 30.6 \pm 5.2 , respectively) (Figure $2 \mathrm{~b}$ and $\mathrm{c}$; one-way ANOVA followed by post hoc Tukey's multiple comparisons test). Moreover, we found colocalization of FG-Fos double-positive neurons with EAAC1, but not GAD67, in the renewal group (Figure 2d). These findings suggest reexposure to the heroin-paired context activates glutamatergic projections from the EC to dDG.

To further clarify the functions of the EC-dDG synaptic transmission in context-induced reinstatement, the excitability of the EC-dDG pathway was examined in vivo using electrophysiology in anesthetized rats immediately after reexposure to context $\mathrm{A}$ or $\mathrm{B}$ for $60 \mathrm{~min}$. It is reported that the PP conveys contextual and spatial information from the EC to dDG (Deng et al, 2010), and potential population spikes (PS) are built by the extracellular summation of action currents during synchronous action potential (AP) firing (Varona et al, 2000). Thus, we recorded the field PS in the $\mathrm{dDG}_{\mathrm{ub}}$ evoked by stimulating the PP. The renewal group showed a significant increase in the PS amplitude and the input-output relationship in the $\mathrm{dDG}_{\mathrm{ub}}$ region. Moreover, high-frequency stimulation at $400 \mathrm{~Hz}$ (Sickmann et al, 2014) evoked larger potentiation in the renewal group that was $37.1 \%$ higher than the control (Figure 2e). The statistical analysis showed a significant interaction between Group and Current $\left(\mathrm{F}_{(4,56)}=4.19, P=0.0049\right)$. These results indicated reexposure to the heroin-paired context results in synaptic potentiation in the EC-dDG pathway.

We performed a DREADD-based strategy (Mahler et al, 2014) to remotely control the EC neurons in vivo and manipulate the EC-dDG pathway. Gi-coupled DREADD ( $A A V_{8}$-hSyn-hM4D (Gi)-mCherry, hM4Di) or a control virus lacking the $D R E A D D$ gene ( $A A V_{5}-S y n-m C h e r r y$, mCherry) was microinjected bilaterally into the EC. On the test day, 5 min before reexposure to context A, CNO (exogenously administered ligand of DREADDs) or vehicle (saline) was microinjected bilaterally into the dDG to inactivate (CNO) $\mathrm{EC}$ terminals in the $\mathrm{dDG}$ or not (saline) (Figure 3a). mCherry had infected majority of cells in the EC, and it was

Figure I Activation of glutamatergic neurons in the dDG mediated context-induced reinstatement of heroin seeking. (a) Overview of the paradigm used in this experiment. (b) The ABA context-induced reinstatement model. Left, number of heroin infusions, active and inactive nose-pokes during the heroin SA

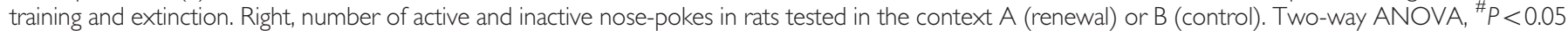

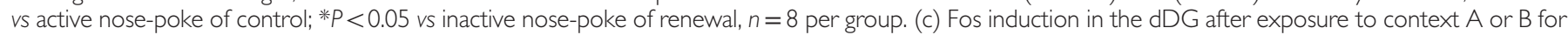

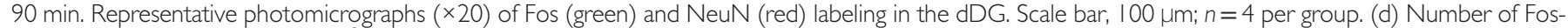

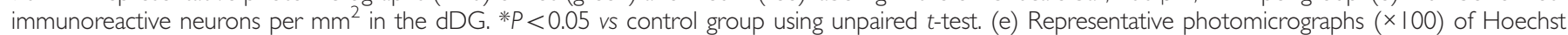

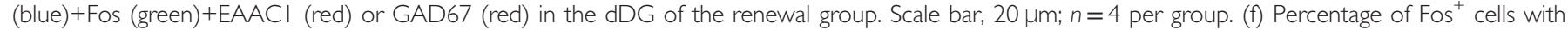

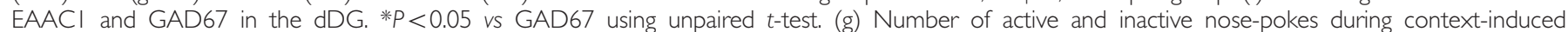

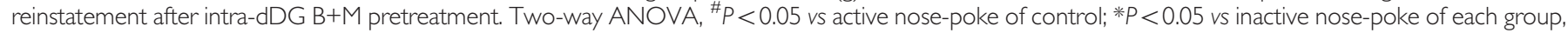

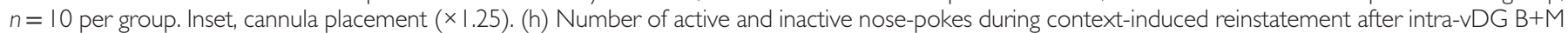

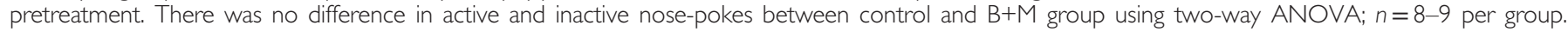

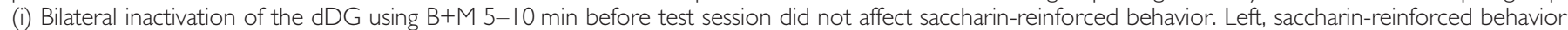

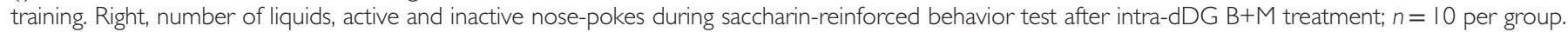
Data are depicted as mean \pm SEM. 
a

dDG
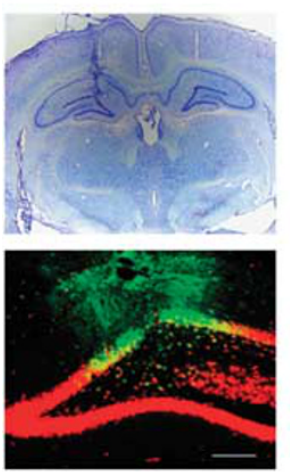

b
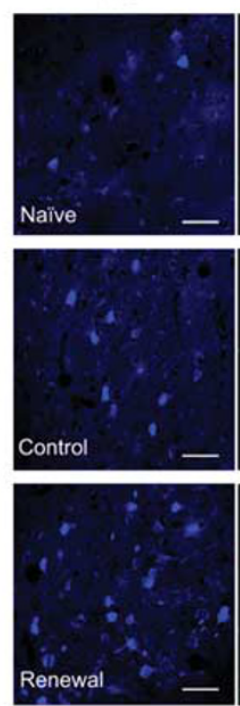

C

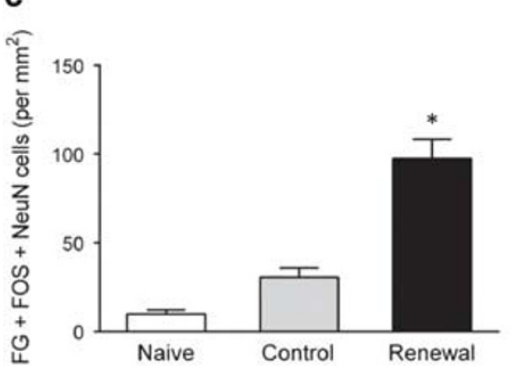

e

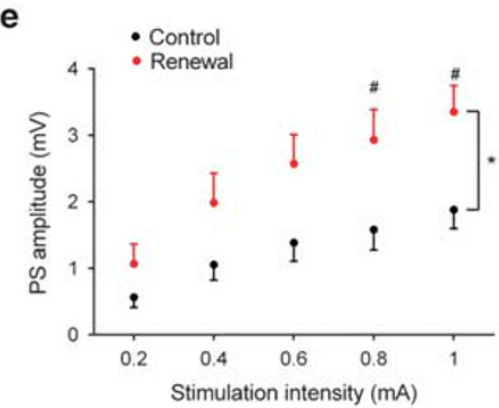

Fos
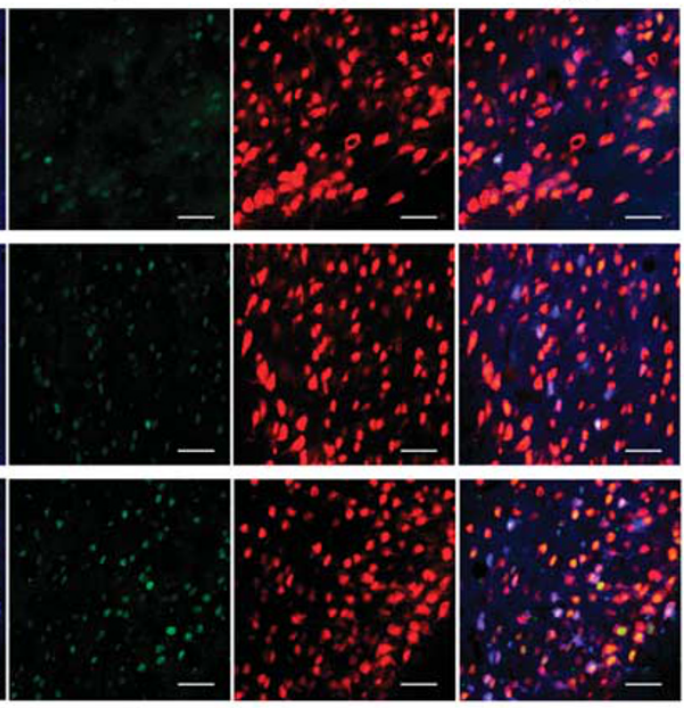

d
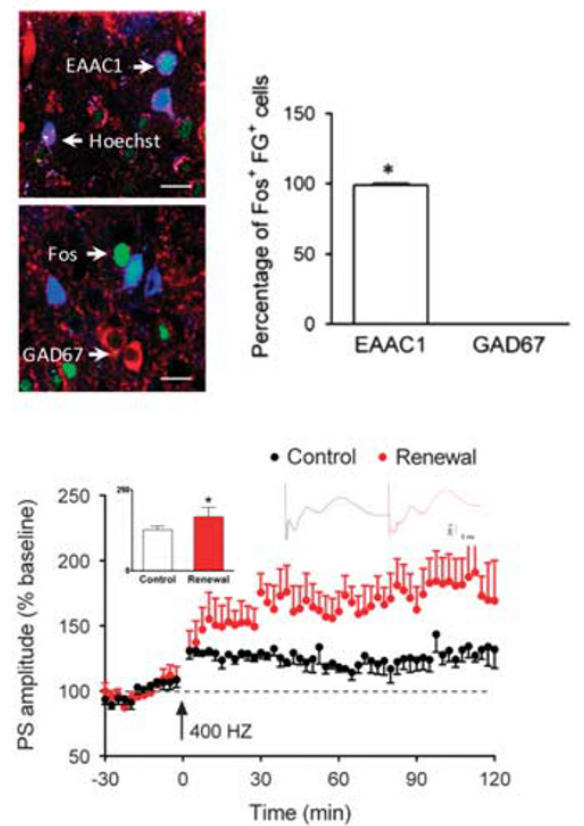

Figure 2 Heroin-paired context exposure induced potentiation of synaptic transmission from the EC to the dDG. (a) FG injection site. Bottom, representative photomicrograph $(\times 10)$ of NeuN (red), FG (blue) in the dDG. (b) Representative photomicrographs ( $\times 40)$ of FG (blue), Fos (green), and NeuN (red) labeling for the EC after exposure to context A, B, or naive rats. Scale bar, $50 \mu \mathrm{m} ; n=4$ per group. (c) Number of FG-Fos double-positive neurons per $\mathrm{mm}^{2}$ in EC. One-way ANOVA, ${ }^{*} P<0.05$ vs naive or control group. (d, left) Representative photomicrographs $(\times 100)$ of FG (blue), Fos (green) + EAACI (red), or GAD67 (red) for the EC of the renewal group. Scale bar, $20 \mu \mathrm{m} ; n=4$ per group. (d, right) Percentage of Fos ${ }^{+}$, FG ${ }^{+}$cells with EAACI and GAD67 in the EC. $* P<0.05$ vs GAD67 using unpaired $t$-test. (e) Long-term potentiation induced using $400 \mathrm{~Hz}$ tetanic stimulation in vivo. Left, input-output curves. Each point represented the PS amplitude in the $\mathrm{dDG}_{\mathrm{ub}}$ from the five individuals for a given stimulus intensity delivered to the PP. Two-way ANOVA, ${ }^{*} P<0.05$ vs control group, ${ }^{\#} P<0.05$ vs each stimulation intensity, $n=8$ per group. Right, high-frequency stimulation at $400 \mathrm{~Hz}$ (arrow) induced a reliable LTP. The amplitude of PS was expressed as a percentage of baseline value. Representative traces of PS amplitude are plotted above the curve. *P $<0.05$ vs control group using unpaired $t$-test; $n=5$ per group. Data are depicted as mean \pm SEM.

also highly restricted to the $\mathrm{dDG}$ where the axons of the EC were located (Figure $3 \mathrm{~b}$ ). CNO pretreatment decreased the number of active nose-pokes by $\sim 60.14 \%$ in rats with hM4Di expression, whereas only $6.67 \%$ decreased in the control group (Figure 3c). The statistical analysis showed a significant interaction between Drug and Virus $\left(\mathrm{F}_{(1,20)}=6.37, P=0.0202\right)$. In contrast, $\mathrm{CNO}$ injections did not alter the number of inactive nose-pokes in the heroinpaired context (Figure 3d), saccharin-reinforced responding (Supplementary Figure S1a), and general locomotor activity (Supplementary Figure S1b).

We also used a classical disconnection approach with $\mathrm{B}+\mathrm{M}$ to inactivate dDG unilaterally, combined with EC contralaterally (contralateral group) or ipsilaterally (ipsilateral 
a

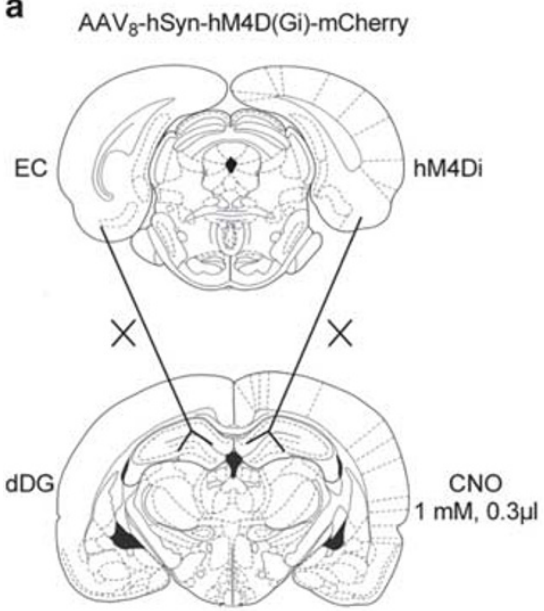

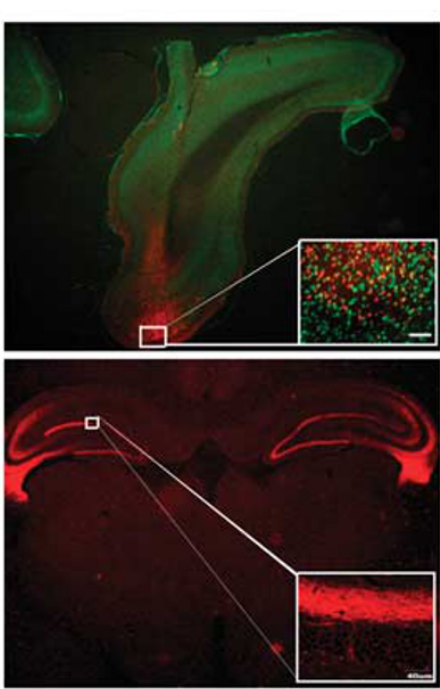

d

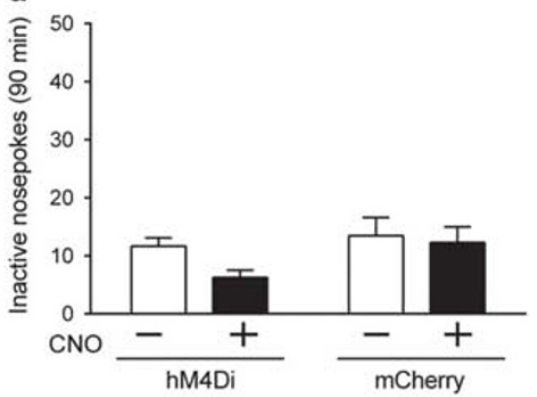

Figure 3 Inhibition of EC-dDG projections decreased context-induced reinstatement of heroin seeking. (a) Projections from the EC to the dDG were transiently inactivated by microinjection of $\mathrm{CNO}$ in the $\mathrm{dDG}$ where the EC axon terminals expressing hM4Di receptors projected. (b) Representative DREADD expression in the experimental groups at 6 weeks after $A A V_{8}-h S y n-h M 4 D$ (Gi)-mCherry microinjected in the EC. Top, typical photomicrographs $(\times 1.25)$ for mCherry-tagged hM4Di (red) and NeuN (green) in the EC. Bottom, Example ( $\times 1.25)$ of axonal labeling for the mCherry-tagged hM4Di receptor (red) in the dDG. (c) Number of active nose-pokes during context-induced reinstatement after intra-dDG vehicle or CNO treatment in animals with hM4Di virus expression or control. Two-way ANOVA, ${ }^{\#} P<0.05$ vs CNO+ of control animals; * $P<0.05$, vs CNO- of hM4Di å; $n=1$ I per group. (d) No difference was observed in inactive nose-pokes during context-induced reinstatement after intra-dDG vehicle or CNO treatment in animals with hM4Di virus expression or control, two-way ANOVA; $n=1$ I per group. Data are depicted as mean \pm SEM.

group). $\mathrm{B}+\mathrm{M}$ contralateral treatment decreased contextinduced reinstatement of heroin seeking (Supplementary Figure S1c). The statistical analysis showed a significant interaction between Group and Nose-Poke $\left(\mathrm{F}_{(2,29)}=8.82\right.$, $P=0.001)$. There was no difference in the control and ipsilateral groups. Taken together, the data in Figures 2 and 3 show that activation of the EC projecting to the $\mathrm{dDG}$ is required for context-induced reinstatement of heroin seeking.

\section{GluN2B-ERK1/2 Signaling Is Downstream of the EC-dDG Glutamatergic Projections in Mediating Context-Induced Reinstatement of Heroin Seeking}

Previous evidence suggests GluN2B in the dDG mediates enhanced plasticity (Ge et al, 2007). Furthermore, Src family kinase activation, NMDAR stimulation, and Src family kinase-mediated GluN2B activation in the dorsal hippocampus play a role in context-induced cocaine-seeking (Xie et al, 2013). We speculated that the projections from the EC to dDG might stimulate dDG neurons via local postsynaptic ionotropic glutamate receptors to promote context-induced reinstatement of heroin seeking. To test this hypothesis, membrane and total protein fractions of ionotropic glutamate receptors in the dDG were measured by western blot. Results demonstrated an upregulation of the GluN2B membrane/total protein ratio (14.7\%) in the renewal group, whereas the levels of other NMDAR subunits (including GluN1 and GluN2A) and AMPAR subunits (including GluA1 and GluA2) were unaffected (Figure 4a and b).

The excitability of NMDARs is known to be regulated by serine/threonine and tyrosine phosphorylation ( $\mathrm{Li}$ et al, 2011; Liu et al, 2008), and tyrosine 1472 residue in GluN2B subunits phosphorylated by Src family kinase promote the surface expression of GluN2B (Zhang et al, 2008). We therefore investigated the phosphorylation level of GluN2B at Y1472 (pY1472 GluN2B) and S1480 (pS1480 GluN2B), the two major sites regulating GluN2B trafficking. Results showed that context-induced reinstatement of heroin seeking elicited a significant increase in pY1472 GluN2B that exhibited a positive correlation with the active nose-pokes behavior in rats during the reinstatement testing, whereas the pS1480 GluN2B level was unaffected (Figure 4c).

It is well documented that phosphorylation of ERK $1 / 2$ is important for preconditioning of response to a wide range of extracellular stimuli, including glutamate stimulation (Yang, 
a

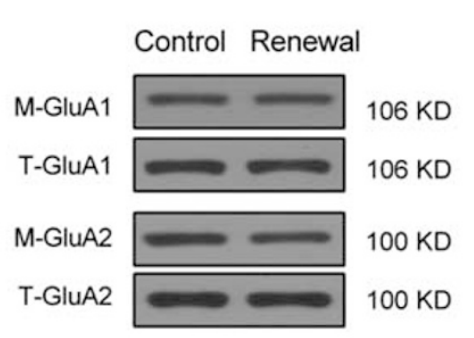

b

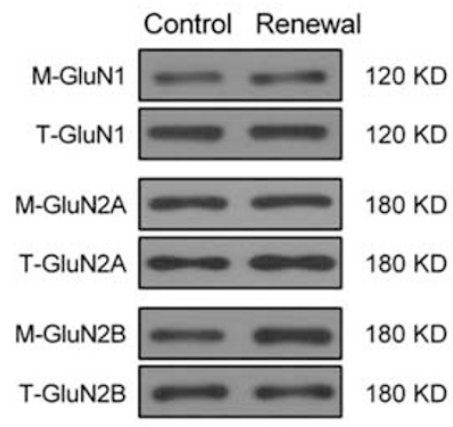

C
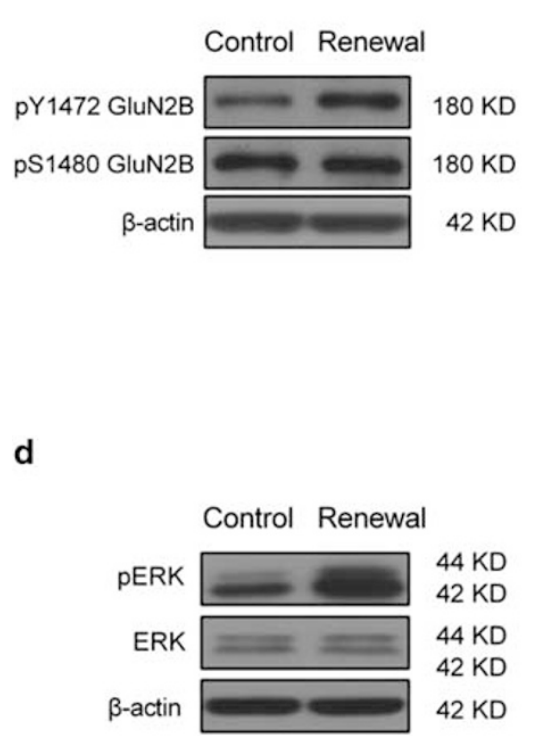
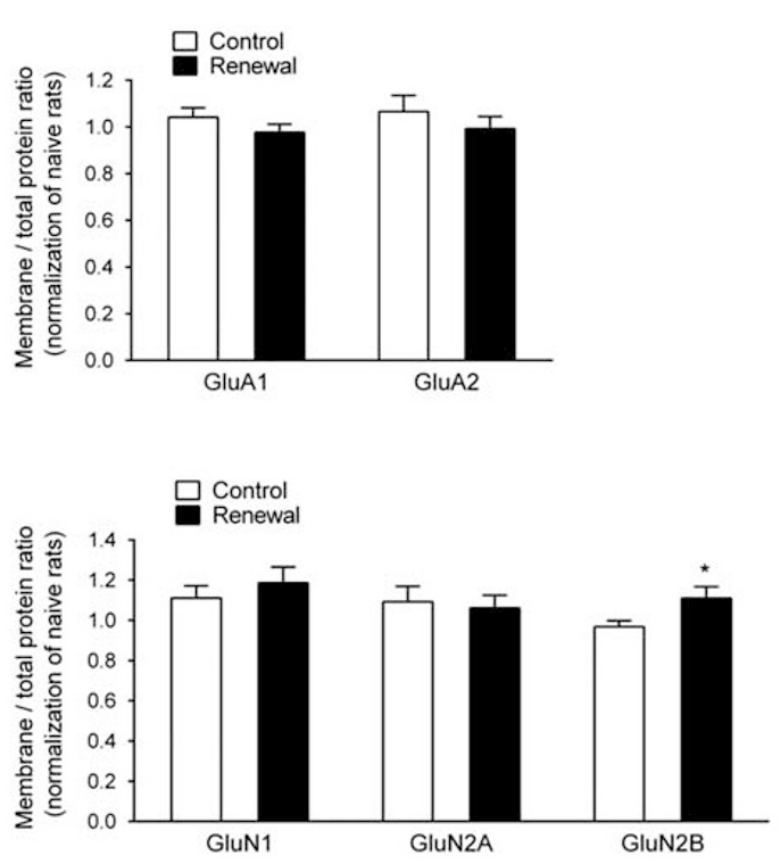
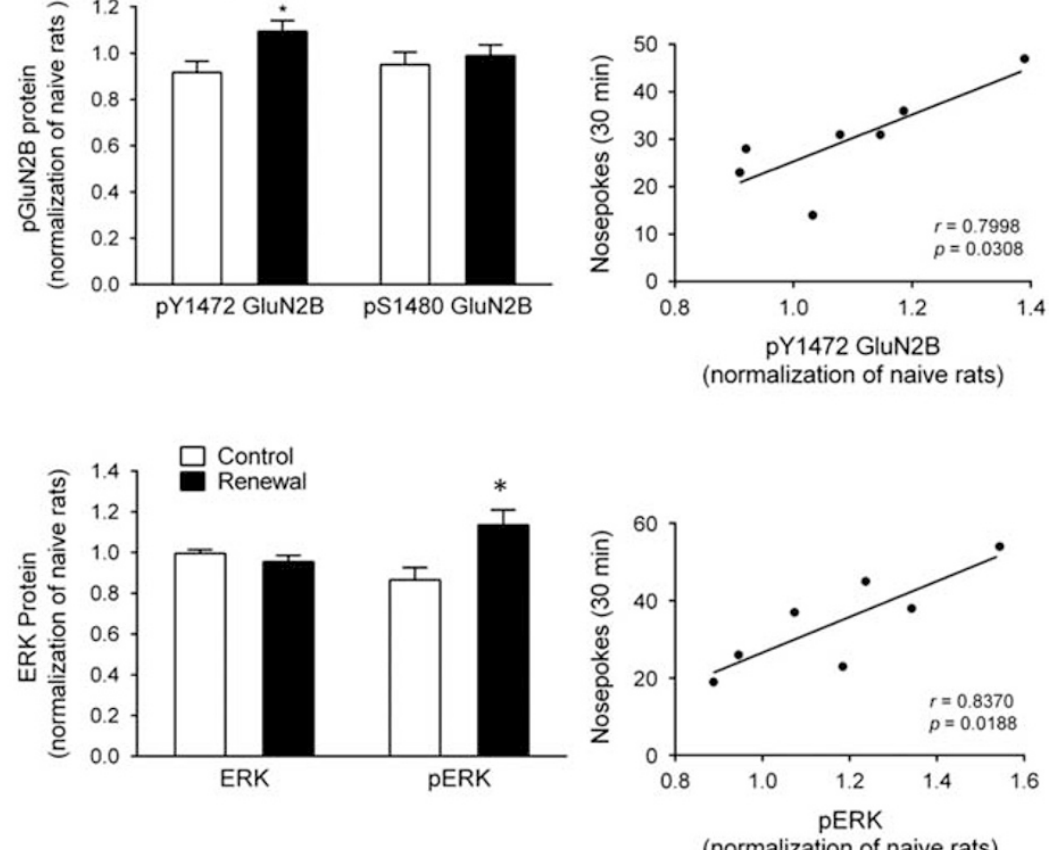

Figure 4 Heroin-paired context reexposure elevated GluN2B-ERKI/2 signaling in the dDG. (a) Western blot analysis of AMPAR subunit GluAI and GluA2 membrane/total protein level in the dDG after 30 min context-induced reinstatement testing. No difference was found between control and renewal group for GluAI and GluA2 subunits using unpaired t-test, respectively; $n=7$ per group. (b) NMDAR subunit GluNI, GluN2A, and GluN2B membrane/total protein level in the dDG after 30 min context-induced reinstatement testing. $* P<0.05$ vs control group using unpaired $t$-test; $n=7$ per group. (c) GluN2B activation in the dDG after context-induced reinstatement testing. Left, western blot analysis of phosphorylation of GluN2B at Y I 472 and SI 480 in the dDG immediately after 30 min context-induced reinstatement testing. ${ }^{*} P<0.05$ vs control group using unpaired t-test. Right, correlation of context-induced reinstatement behavior with pY I472 GluN2B activation in the renewal group; $n=7$ per group. (d) ERKI/2 and pERKI/2 protein level in the dDG after 30 min context-induced reinstatement testing. Left, western blot analysis of phosphorylation of ERKI/2 and pERKI/2 in the dDG immediately after context-induced reinstatement testing. $* P<0.05$ vs control group using unpaired $t$-test. Right, correlation of context-induced reinstatement behavior with PERKI/2 activation in the renewal group; $n=7$ per group. Data are depicted as mean \pm SEM.

2004), and it is regulated by GluN2B (Yang et al, 2015). Therefore, we examined the activity of pERK1/2 after reinstatement testing. Results showed that context-induced reinstatement of heroin seeking increased the phosphorylation of ERK1/2 at T202 and Y204 (pERK) that is positively correlated with the reinstatement behavior. There was no difference in the level of total ERK1/2 (Figure 4d). These results indicate that context-induced 
a

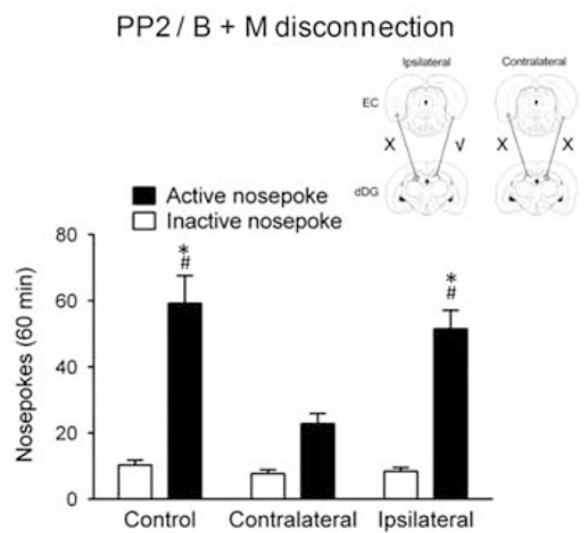

C

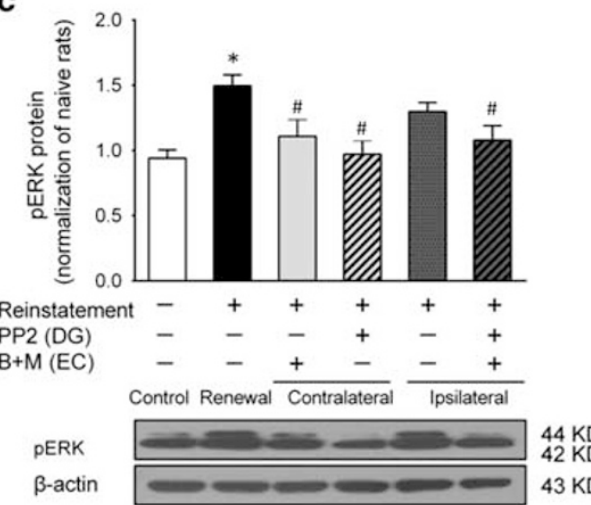

d

RO25-6981 / B + M disconnection

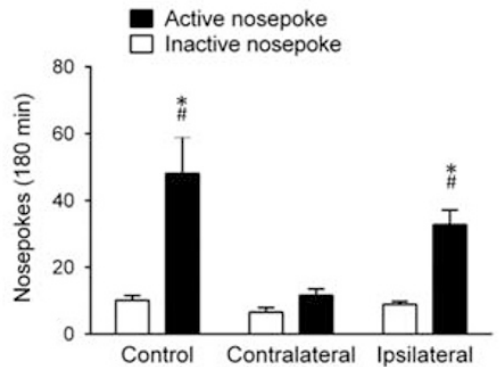

b
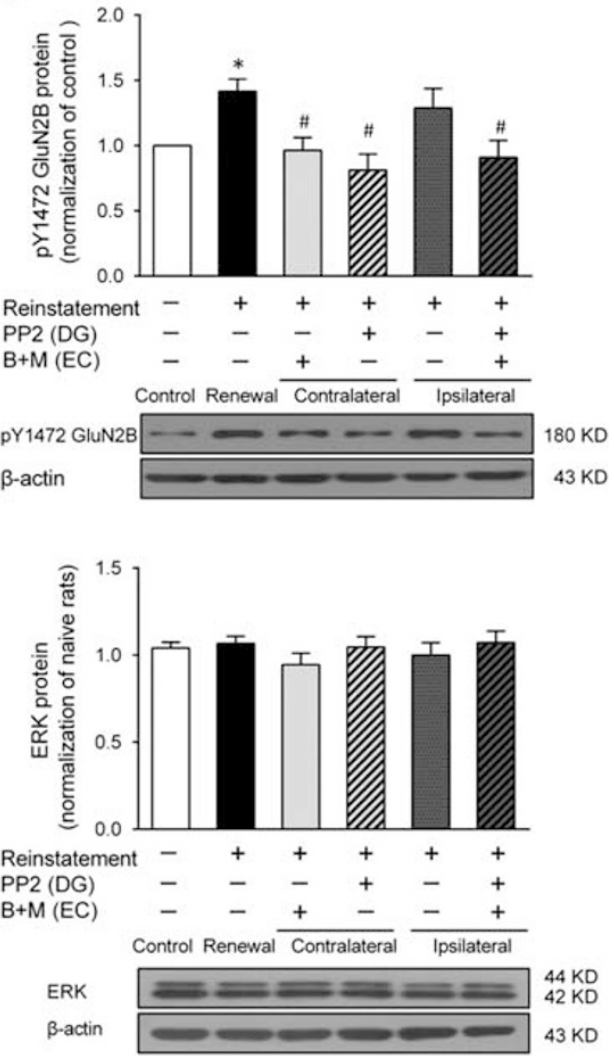

e

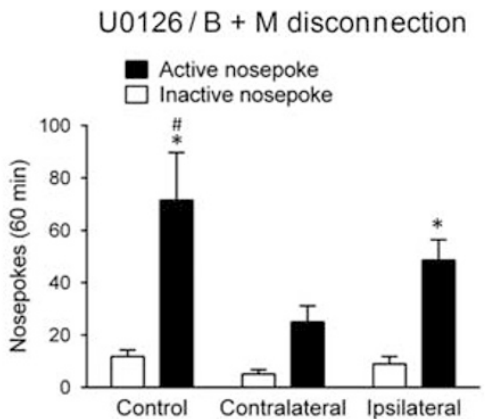

Figure 5 Inhibition of EC-dDG projections via GluN2B-ERKI/2 signaling decreased context-induced reinstatement of heroin seeking. (a) Number of nosepokes during context-induced reinstatement under PP2 and B+M disconnection treatment. Two-way ANOVA, ${ }^{*} P<0.05$ vs active nose-poke of contralateral disconnection animals; $* P<0.05$, vs inactive nose-poke of each group, $n=10$ per group. Insert, overview of the paradigm used in this experiment. (b) $p Y \mid 472$ GluN2B level in the dDG after 30 min context-induced reinstatement under the PP2 and B+M disconnection treatment. *P $<0.05$ vs control group using unpaired $t$-test. One-way ANOVA, ${ }^{\#} P<0.05$ vs renewal group, $n=7$ per group. (c) $p E R K I / 2$ and ERKI/2 protein level in the dDG after 30 min contextinduced reinstatement under the $\cup 0126$ and $B+M$ disconnection treatment. ${ }^{*} P<0.05$ vs control group using unpaired $t$-test. One-way ANOVA, ${ }^{\#} P<0.05$ vs renewal group, $n=5-7$ per group. (d) Number of nose-pokes during context-induced reinstatement under RO25-698I and B+M disconnection treatment. Two-way ANOVA, ${ }^{\#} P<0.05$ vs active nose-poke of contralateral disconnection animals; ${ }^{*} P<0.05$, vs inactive nose-poke of each group, $n=9$ per group. (e) Number of nose-pokes during context-induced reinstatement under $\mathrm{U} 0 \mathrm{I} 26$ and B+M disconnection treatment. Two-way ANOVA, \#P<0.05 vs active nosepoke of contralateral disconnection group; $* P<0.05$ vs inactive nose-poke of each group, $n=7$ per group. Data are depicted as mean \pm SEM.

reinstatement of heroin seeking is associated with an increase in GluN2B activation at Y1472, GluN2B membrane protein expression, and subsequent activation of ERK1/2 signaling in the dDG.

The $\mathrm{dDG}$ receives the projections from the EC. To determine whether GluN2B-ERK1/2 signaling in the dDG is necessary for context-induced reinstatement of heroin seeking, we performed a modified disconnection procedure to interfere with the interaction between glutamatergic projections from the $\mathrm{EC}$ to $\mathrm{dDG}$ via local GluN2B postsynaptic receptors. As shown in Figure 5, microinfusions of $\mathrm{PP} 2$ into the unilateral $\mathrm{dDG}$ and $\mathrm{B}+\mathrm{M}$ into the contralateral EC (contralateral group) significantly decreased context-induced reinstatement by $61.5 \%$ compared with the control group (saline into the unilateral dDG and ipsilateral/ contralateral EC), and by $55.7 \%$ compared with the ipsilateral group (PP2 into the unilateral $\mathrm{dDG}$ and $\mathrm{B}+\mathrm{M}$ into the ipsilateral EC) (Figure 5a). The statistical analysis 
showed a significant interaction between Group and NosePoke $\left(\mathrm{F}_{(2,27)}=9.15, P=0.0009\right)$. This disconnection procedure had no influence on saccharin-reinforced responding, general locomotor activity, and lever presses in the extinction context (Supplementary Figure S2). In addition, western blot analysis showed PP2 and $\mathrm{B}+\mathrm{M}$ contralateral disconnection pretreatment reversed the increase in pY1472 GluN2B (Figure 5b) and downstream pERK1/2 induced by contextinduced reinstatement of heroin seeking, without altering the total ERK1/2 protein level in the dDG (Figure $5 \mathrm{c}$ ).

These results suggest ERK1/2 is downstream of the GluN2B and is regulated by the activation of GluN2B at Y1472. Similarly, unilateral intra-dDG RO25-6981 combined with intra-EC $\mathrm{B}+\mathrm{M}$ pretreatment contralaterally decreased context-induced reinstatement (Figure 5d). The statistical analysis showed a significant interaction between Group and Nose-Poke $\left(\mathrm{F}_{(2,24)}=5.83, P=0.0087\right)$. Moreover, U0126/ B $+\mathrm{M}$ disconnection also decreased context-induced reinstatement (Figure 5e). The statistical analysis showed significant effects of Group $\left(\mathrm{F}_{(2,18)}=4.40, P=0.0278\right)$ and Nose-Poke $\left(\mathrm{F}_{(1,18)}=34.72, P<0.05\right)$.

Taken together, glutamatergic projections from the EC to dDG modulate context-induced reinstatement of heroin seeking in which Src family kinase-mediated activation of GluN2B-ERK1/2 signaling in the $\mathrm{dDG}$ is required.

\section{DISCUSSION}

Our study demonstrates the EC-dDG pathway is activated by, and necessary for, context-induced reinstatement of heroin seeking in rats, and it depends on GluN2B activation at Y1472 and subsequent ERK1/2 signaling in the dDG. Functional disconnection using Gi-coupled DREADDs to specifically inactivate the EC-dDG pathway and pharmacological disconnection to inactivate the EC, combined with GluN2B-ERK1/2 signaling in the contralateral dDG, both decreased context-induced reinstatement of heroin seeking. These manipulations did not affect nose-poke responding in the extinction context, saccharin-reinforced responding, and general locomotor activity.

\section{The EC-dDG Pathway Mediates Context-Induced Heroin Seeking}

Previous research investigated the role of the EC and DG in contextual and spatial memory. Same as the DG, the EC is also involved in contextual fear memory reconsolidation and extinction (Baldi and Bucherelli, 2014). Hargreaves et al (2005) reported that EC neurons projecting to the dorsal hippocampus display robust and selective firing in a rewardassociated environment. In addition, the DG receives glutamatergic input from the EC through the PP that conveys contextual information from the EC to DG (Deng et al, 2010). Our results shed light on the function of the EC-dDG pathway in context-induced reinstatement of heroin seeking via the role of this pathway in the retrieval of drug-paired contextual memory. We speculate that inactivation of the EC-dDG pathway would inhibit the retrieval of the learned association between heroin effects and heroin-paired contexts, leading to prevent drug relapse. Of note, EC-dDG silencing that decreased context-induced reinstatement of heroin seeking was not because of the inability to nose-poke or motor deficits, as these manipulations did not alter saccharin-reinforced responding and general locomotor activity in rats.

\section{Anatomical Segregation and Function of EC and dDG}

The EC is divided into the medial EC and lateral EC (MEC and LEC). Previous evidence supports a role for the MEC in spatial processing and for the LEC in nonspatial information (Hafting et al, 2005; Hargreaves et al, 2005). However, some studies questioned this functional segregation. Van Cauter et al (2013) reported that the LEC is involved in the joint processing of spatial and nonspatial information, whereas other studies demonstrated that MEC and LEC both contribute to contextual memory (Hunsaker et al, 2013; Majchrzak et al, 2006). The LEC provides information with external sensory content, and the MEC provides the spatial context, with both combining to encode contextual memory (Neunuebel et al, 2013). In our study, heroin-paired context is a complex stimulus that contains spatial and sensory (visual, auditory, tactile, olfactory sense) information. Moreover, the retrograde tracing experiments showed the FG microinfused in the dDG was observed in both the LEC and MEC after reexposure to the heroin-paired context, suggesting that both LEC and MEC contribute to the retrieval of heroin contextual memories. Our finding gets a conclusion consistent with previous reports that both MEC and LEC contribute to contextual memory (Hunsaker et al, 2013; Majchrzak et al, 2006). Consistent with its proposed roles in both cognitive and emotional domains, the hippocampus shows marked variation along its dorsoventral axis in terms of both afferent and efferent connectivity (Fanselow and Dong, 2010). The dorsal hippocampus projects extensively to associational cortical regions, whereas the ventral hippocampus projects to regions implicated in autonomic, neuroendocrine, and motivational responses to emotionally charged stimuli (Moser and Moser, 1998). Previous studies showed that granule cells in the $\mathrm{dDG}$ selectively control the encoding of contextual memories, whereas those in the vDG do not (Kheirbek et al, 2013). This pattern of results is consistent with our results: inactivation of neural activity in dDG but not vDG decreased context-induced reinstatement of heroin seeking.

\section{The EC-dDG Pathway Influences Context-Induced Reinstatement of Heroin Seeking Via Local Postsynaptic GluN2B-ERK1/2 Signaling}

Context-induced reinstatement of heroin seeking significantly activated the GluN2B-ERK 1/2 signaling, indicating phosphorylation of GluN2B at Y1472 accelerates GluN2B trafficking, resulting in the increase of GluN2B surface expression and the activation of ERK1/2 signaling. These data extend results from a previous study that showed that Src family kinase-mediated GluN2B activation in the dorsal hippocampus is necessary for context-induced reinstatement of cocaine seeking (Xie et al, 2013). The dDG NMDARs can rapidly encode unique memory of a context and discriminate it from other similar contexts previously encountered (McHugh et al, 2007). In addition, GluN2B signaling mediates the enhanced synaptic plasticity in the dDG cells 
(Ge et al, 2007). In the disconnection procedure using in our study, pY1472 GluN2B and downstream pERK1/2 protein levels were decreased in the $\mathrm{dDG}$ not only when the $\mathrm{dDG}$ local received PP2 microinfusions, but also when we inactivated the $\mathrm{EC}$ neurons with $\mathrm{B}+\mathrm{M}$. These data suggest that ERK1/2 is the downstream of GluN2B that is regulated by $\mathrm{pY} 1472$ GluN2B in the $\mathrm{dDG}$, and the local postsynaptic GluN2B-ERK $1 / 2$ signaling in the $\mathrm{dDG}$ is regulated by the EC. In summary, our results indicate that GluN2B-ERK1/2 signaling in the $\mathrm{dDG}$ is mediated by upstream EC activation, and pERK1/2 is the downstream signal of pY1472 GluN2B.

Taken together, our data demonstrate that in the dDG local postsynaptic Src family kinase-mediated GluN2BERK1/2 signaling is necessary for context-induced reinstatement of heroin seeking.

In conclusion, the present study provides new evidence that the glutamatergic projection from the $\mathrm{EC}$ to $\mathrm{dDG}$ is activated during, and is necessary for, context-induced reinstatement of heroin seeking, in which the activation of local postsynaptic GluN2B-ERK1/2 signaling is required. These findings not only confirm the function of the EC-dDG pathway in drug-paired contextual memory, but also highlight a new mechanism of context-induced reinstatement of heroin seeking.

\section{FUNDING AND DISCLOSURE}

The authors declare no conflict of interest.

\section{ACKNOWLEDGMENTS}

This work was supported by the National Natural Science Foundation (grant number 81471353), the National Basic Research Program (grant number 2015CB553500), and Science Fund for Creative Research Groups from the National Natural Science Foundation (81521063) of China to C Cui.

\section{REFERENCES}

Baldi E, Bucherelli C (2014). Entorhinal cortex contribution to contextual fear conditioning extinction and reconsolidation in rats. Neurobiol Learn Mem 110: 64-71.

Bossert JM, Adhikary S, St. Laurent R, Marchant NJ, Wang H, Morales $\mathrm{M}$ et al (2016). Role of projections from ventral subiculum to nucleus accumbens shell in context-induced reinstatement of heroin seeking in rats. Psychopharmacology 233: 1991-2004.

Bossert JM, Stern AL, Theberge FR, Cifani C, Koya E, Hope BT et al (2011). Ventral medial prefrontal cortex neuronal ensembles mediate context-induced relapse to heroin. Nat Neurosci 14: 420-422.

Bossert JM, Stern AL, Theberge FRM, Marchant NJ, Wang HL, Morales $M$ et al (2012). Role of projections from ventral medial prefrontal cortex to nucleus accumbens shell in context-induced reinstatement of heroin seeking. J Neurosci 32: 4982-4991.

Carretero-Guillen A, Pacheco-Calderon R, Delgado-Garcia JM, Gruart A (2013). Involvement of hippocampal inputs and intrinsic circuit in the acquisition of context and cues during classical conditioning in behaving rabbits. Cereb Cortex 25: $1278-1289$.

Crombag HS, Bossert JM, Koya E, Shaham Y (2008). Contextinduced relapse to drug seeking: a review. Philos Trans $R$ Soc $B$ Biol Sci 363: 3233-3243.
Deng W, Aimone JB, Gage FH (2010). New neurons and new memories: how does adult hippocampal neurogenesis affect learning and memory? Nat Rev Neurosci 11: 339-350.

Fanselow MS, Dong HW (2010). Are the dorsal and ventral hippocampus functionally distinct structures? Neuron 65: 7-19.

Ge S, Yang CH, Hsu KS, Ming GL, Song H (2007). A critical period for enhanced synaptic plasticity in newly generated neurons of the adult brain. Neuron 54: 559-566.

Hafting T, Fyhn M, Molden S, Moser MB, Moser EI (2005). Microstructure of a spatial map in the entorhinal cortex. Nature 436: 801-806.

Hargreaves EL, Rao G, Lee I, Knierim JJ (2005). Major dissociation between medial and lateral entorhinal input to dorsal hippocampus. Science 308: 1792-1794.

Hernandez-Rabaza V, Hontecillas-Prieto L, Velazquez-Sanchez C, Ferragud A, Perez-Villaba A, Arcusa A et al (2008). The hippocampal dentate gyrus is essential for generating contextual memories of fear and drug-induced reward. Neurobiol Learn Mem 90: 553-559.

Hu L, Jing X, Cui C, Xing G, Zhu B (2014). NMDA receptors in the midbrain play a critical role in dopamine-mediated hippocampal synaptic potentiation caused by morphine. Addict Biol 19: 380-391.

Hunsaker MR, Chen V, Tran GT, Kesner RP (2013). The medial and lateral entorhinal cortex both contribute to contextual and item recognition memory: a test of the binding of items and context model. Hippocampus 23: 380-391.

Kaufman DL, McGinnis JF, Krieger NR, Tobin AJ (1986). Brain glutamate decarboxylase cloned in lambda gt-11: fusion protein produces gamma-aminobutyric acid. Science 232: $1138-1140$.

Keiflin R, Reese RM, Woods CA, Janak PH (2013). The orbitofrontal cortex as part of a hierarchical neural system mediating choice between two good options. I Neurosci 33: 15989-15998.

Kheirbek MA, Drew LJ, Burghardt NS, Costantini DO, Tannenholz L, Ahmari SE et al (2013). Differential control of learning and anxiety along the dorsoventral axis of the dentate gyrus. Neuron 77: 955-968.

Kim JJ, Fanselow MS (1992). Modality-specific retrograde amnesia of fear. Science 256: 675-677.

Knierim JJ, Neunuebel JP, Deshmukh SS (2014). Functional correlates of the lateral and medial entorhinal cortex: objects, path integration and local-global reference frames. Philos Trans $R$ Soc Lond B Biol Sci 369: 20130369.

Lammel S, Lim BK, Ran C, Huang KW, Betley MJ, Tye KM et al (2012). Input-specific control of reward and aversion in the ventral tegmental area. Nature 491: 212-217.

Li S, Cao J, Yang X, Suo Z, Shi L, Liu Y et al (2011). NR2B phosphorylation at tyrosine 1472 in spinal dorsal horn contributed to N-methyl-D-aspartate-induced pain hypersensitivity in mice. J Neurosci Res 89: 1869-1876.

Liu XJ, Gingrich JR, Vargas-Caballero M, Dong YN, Sengar A, Beggs $S$ et al (2008). Treatment of inflammatory and neuropathic pain by uncoupling Src from the NMDA receptor complex. Nat Med 14: 1325-1332.

Mahler SV, Vazey EM, Beckley JT, Keistler CR, McGlinchey EM, Kaufling J et al (2014). Designer receptors show role for ventral pallidum input to ventral tegmental area in cocaine seeking. Nat Neurosci 17: 577-585.

Majchrzak M, Ferry B, Marchand AR, Herbeaux K, Seillier A, Barbelivien A (2006). Entorhinal cortex lesions disrupt fear conditioning to background context but spare fear conditioning to a tone in the rat. Hippocampus 16: 114-124.

McHugh TJ, Jones MW, Quinn JJ, Balthasar N, Coppari R, Elmquist JK et al (2007). Dentate gyrus NMDA receptors mediate rapid pattern separation in the hippocampal network. Science 317: 94-99. 
Moser MB, Moser EI (1998). Functional differentiation in the hippocampus. Hippocampus 8: 608-619.

Neunuebel JP, Yoganarasimha D, Rao G, Knierim JJ (2013). Conflicts between local and global spatial frameworks dissociate neural representations of the lateral and medial entorhinal cortex. J Neurosci 33: 9246-9258.

O'Brien CP, Childress AR, McLellan AT, Ehrman R (1992). Classical conditioning in drug-dependent humans. Ann NY Acad Sci 654: 400-415.

Ramirez DR, Bell GH, Lasseter HC, Xie X, Traina SA, Fuchs RA (2009). Dorsal hippocampal regulation of memory reconsolidation processes that facilitate drug context-induced cocaineseeking behavior in rats. Eur J Neurosci 30: 901-912.

Redondo RL, Kim J, Arons AL, Ramirez S, Liu X, Tonegawa S (2014). Bidirectional switch of the valence associated with a hippocampal contextual memory engram. Nature 513: 426-430.

Rivera PD, Raghavan RK, Yun S, Latchney SE, McGovern M, García EF et al (2015). Retrieval of morphine-associated context induces cFos in dentate gyrus neurons. Hippocampus 25: 409-414.

Shen F, Wang X, Ge F, Li Y, Cui C (2016). Essential role of the NO signaling pathway in the hippocampal CA1 in morphineassociated memory depends on glutaminergic receptors. Neuropharmacology 102: 216-228.

Sickmann HM, Patten AR, Morch K, Sawchuk S, Zhang C, Parton R et al (2014). Prenatal ethanol exposure has sex-specific effects on hippocampal long-term potentiation. Hippocampus 24: 54-64.

Van Cauter T, Camon J, Alvernhe A, Elduayen C, Sargolini F, Save E (2013). Distinct roles of medial and lateral entorhinal cortex in spatial cognition. Cereb Cortex 23: 451-459.

Varona P, Ibarz JM, Lopez-Aguado L, Herreras O (2000). Macroscopic and subcellular factors shaping population spikes. J Neurophysiol 83: 2192-2208.

Weiner LP (ed) (2008). Neural Stem Cell: Methods and Protocols, 2nd edn. Humana: Totowa, NJ, 406pp.

Wikler A (1973). Dynamics of drug dependence. Implications of a conditioning theory for research and treatment. Arch Gen Psychiatry 28: 611-616.
Xie X, Arguello AA, Wells AM, Reittinger AM, Fuchs RA (2013). Role of a hippocampal SRC-family kinase-mediated glutamatergic mechanism in drug context-induced cocaine seeking. Neuropsychopharmacology 38: 2657-2665.

Xie X, Ramirez DR, Lasseter HC, Fuchs RA (2010). Effects of mGluR1 antagonism in the dorsal hippocampus on drug contextinduced reinstatement of cocaine-seeking behavior in rats. Psychopharmacology (Berl) 208: 1-11.

Xu Y, Lv X, Cui C, Ge F, Li Y, Zhang H (2012). Essential role of NR2B-containing NMDA receptor-ERK pathway in nucleus accumbens shell in morphine-associated contextual memory. Brain Res Bull 89: 22-30.

Yang JX, Hua L, Li YQ, Jiang YY, Han D, Liu H et al (2015). Caveolin-1 in the anterior cingulate cortex modulates chronic neuropathic pain via regulation of NMDA receptor $2 \mathrm{~B}$ subunit. J Neurosci 35: 36-52.

Yang L (2004). A novel Ca2+-independent signaling pathway to extracellular signal-regulated protein kinase by coactivation of NMDA receptors and metabotropic glutamate receptor 5 in neurons. J Neurosci 24: 10846-10857.

Zhang S, Edelmann L, Liu J, Crandall JE, Morabito MA (2008). Cdk5 regulates the phosphorylation of tyrosine 1472 NR2B and the surface expression of NMDA receptors. J Neurosci 28: 415-424.

cc) (i) () $\ominus$ This work is licensed under a Creative Commons Attribution-NonCommercial-NoDerivs $\quad 4.0$ International License. The images or other third party material in this article are included in the article's Creative Commons license, unless indicated otherwise in the credit line; if the material is not included under the Creative Commons license, users will need to obtain permission from the license holder to reproduce the material. To view a copy of this license, visit http://creativecommons.org/licenses/by-nc-nd/4.0/

(C) The Author(s) 2017

Supplementary Information accompanies the paper on the Neuropsychopharmacology website (http://www.nature.com/npp) 